\title{
Study of Cloud Computing
}

\author{
Drashti Hirani ${ }^{1}$, Darshan Thoria ${ }^{2}$ \\ Drashti Hirani is currently pursuing masters degree program in Computer Science \& Engineering in Gujarat \\ Technological University, India \\ Darshan Thoria is currently pursuing masters degree program in Computer Engineering in Gujarat Technologi- \\ cal University, India.
}

\begin{abstract}
Cloud computing is rising as the next generation platform for computation. May be in future Cloud computing will be the main platform to save the world; this makes people can have everything they need on it. Main advantages of the Cloud computing is used for on-demand gathering of information, technology services and products. The name Cloud has come from the Internet, based on how it is depicted in computer network diagrams, and is an abstraction for the complex infrastructure it conceals. In general we can define cloud computing is style of computing in which IT-related capabilities are provided "as a service", allowing users to access technology-enabled services from the Internet without knowledge of, expertise with, or control over the technology infrastructure that supports them. Email application was probably the first service on the "cloud".

KeyWords: Cloud Computing, Virtulization, Utility Computing.
\end{abstract}

\section{Introduction}

The cloud has reached into our daily life and led to a broader range of innovations, but people often misunderstand what cloud computing is. Built on many old IT technologies, cloud computing is actually an evolutionary approach that completely changes how computing services are produced, priced and delivered. It allows to access services that reside in a distant datacenter, other than local computers or other Internet-connected devices. Cloud services are charged according to the amount consumed by worldwide users. Such an idea of computing as a utility is a long-held dream in the computer industry, but it is still immature until the advent of lowcost datacenters that will enable this dream to come true.

Datacenters, behaving as "cloud providers", are computing infrastructures which provide many kinds of agile and effective services to customers. A wide range of IT companies including Amazon, Cisco, Yahoo, Sales force, Face book, Microsoft and Google have their own datacenters and provide pay-as-you-go cloud services. Two different but related types of cloud service should be distinguished first. One is on-demand computing instance, and the other is on-demand computing capacity. Equipped with similar machines, datacenters can scale out by providing additional computing instances, or can support data- or compute-intensive applications via scaling capacity.

Amazon's EC2 and Eucalyptus are examples of the first category, which provides computing instances according to needs. Google and Yahoo belong to the second category. In these datacenters, the need of processing large amounts of raw data is primarily met with distributed and parallel computing, and the data can be moved from place to place and assigned changing attributes based on its lifecycle, requirements, and usefulness.

The above two types of cloud services classify cloud computing into two distinct deployment models: public and private. A public cloud is designed to provide cloud services to a variety of third-party clients who use the same cloud resources. Public cloud services such as Google's App Engine are open to anyone at anytime and anywhere. On the contrary, a private cloud is devoted to a single organization's internal use. Google, for example, uses GFS,MapReduce, and BigTable as part of its private cloud services, so these services are only open inside the enterprise. It's important to note that Google uses its private cloud to provide public cloud services, such as productive applications, media delivery, and social interaction.

\section{Cloud definations}

I. Foster: "A large-scale distributed computing paradigm that is driven by economies of scale, in which a pool of abstracted virtualized, dynamically-scalable, managed computing power, storage, platforms, and services are delivered on demand to external customers over Internet."

Gartner : "A style of computing where scalable and elastic IT capabilities are provided as a service to multiple external customers using Internet technologies."

NIST: "Cloud computing is a model for enabling convenient, on-demand network access to a shared pool of 
configurable computing resources (e.g., networks, servers, storage, applications, and services) that can be rapidly provisioned and released with minimal management effort or service provider interaction."

\section{Challenges and motivations}

Cloud computing is still in its infancy, but it has presented new opportunities to users and deve -opers who can benefit from economies of scales, commoditization of assets and conformance to programming standards. Its attributes such as scalability, elasticity, low barrier to entry and a utility type of delivery make this new paradigm quickly marketable.

The illusion of scalability is bounded by the limitations that cloud providers place on their clients. Resource limits are exposed at peak conditions of the utility itself. For example, bursting spring festival messages lead to outage for telecom operators, so they have to set limits on the number of short messages before New Year Eve. The same problem appears in cloud computing. These outages will happen on peak computing days such as the day when Internet Christmas sales traditionally begin.

Another illusion of elasticity is affected by an inconsistent pricing scheme that makes the investment no longer scalable to its payoffs. The price for extra large instance might be nonlinear to its size, compared with the price for standard instances. Moreover, the low barrier to entry can also be accompanied by a low barrier to provisioning.

Based on the above analysis, resource management is a topic worthy of investigation, and is a key issue to decide whether the new computing paradigm can be adopted more and obtain great business success.

From the public perspective of a cloud datacenter, its goal is reducing cost and maximizing its profit since the public cloud plays a role of service provider in a real market. The resource management will focus on pricing schemes to ensure economic benefits for the cloud agents.

From the private perspective of a cloud datacenter, it focuses more on the system performance of the datacenter. In that case, improvement from resource management mainly concerns technical issues. For example, it is important to optimize the scheduling schemes to reduce job completion time and to improve resource utilization, when many MapReduce jobs are running in parallel at the same time.

\section{Objectives and contributions}

These thesis studies resource management problems related to cloud computing, such as resource allocation, scheduling and simulation. The major contributions are as follows.

A survey of current trends and research opportunities in cloud computing:. We investigate the state-of-theart efforts on cloud computing, from both industry and academic standpoints. Through comparison with other related technologies and computing paradigms, we identify several challenges from the cloud adoption perspective. We also highlight the resource management issue that deserves substantial research and development.

A cloud scheduling hierarchy to distinguish different requirements of cloud services:. We systemize the scheduling problem in cloud computing, and present a cloud scheduling hierarchy, mainly splitting into userlevel and system-level.

Cloud computing is a model for enabling convenient, on-demand network access to a shared pool of configurable computing resources (e.g., networks, servers, storage, applications, and services) that can be rapidly provisioned and released with minimal management effort or service provider interaction."

\section{System architecture}

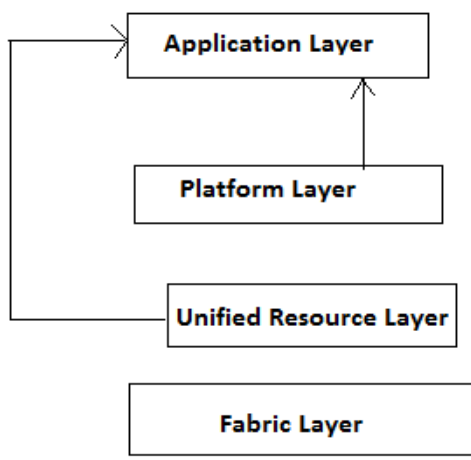

Figure 1 - System architecture 
Clouds are usually referred to as a large pool of computing and storage resources, which can be accessed via standard protocols with an abstract interface . A four-layer architecture for cloud computing is shown in Figure 1 .

The fabric layer contains the raw hardware level resources, such as compute resources,storage resources, and network resources. On the unified resource layer, resources have been virtualized so that they can be exposed to upper layer and end users as integrated resources. The platform layer adds on a collection of specialized tools, middleware and services on top of the unified resources to provide a development and deployment platform. The application layer includes the applications that would run in the clouds.

\section{Cloud evolution}

Although the idea of cloud computing is not new, it has rapidly become a new trend in the information and communication technology domain and gained significant commercial success over past years. No one can deny that cloud computing will a play pivotal role in the next decade. Why cloud computing emerges now, not before? This section looks back on the development history of cloud computing.

\section{Getting ready for cloud}

- Datacenter: Even faster than Moore's law, the number of servers and datacenters has increased dramatically in past few years. Datacenter has become the reincarnation of the mainframe concept. It is easier to build a largescale commodity-computer datacenter than ever before, just gathering these building blocks together on a parking lot and plugging them into the Internet.

- Internet: Recently, network performance has improved rapidly. Wired, wireless and $4^{\text {th }}$ generation mobile communication make Internet available to most of the planet. Cities and towns are wired with hotspots. The transportation such as air, train, or ship also equips with satellite based wi-fi or undersea fiber-optic cable. People can connect to the Internet anywhere and at anytime. The universal, high-speed, broadband Internet lays the foundation for the widespread applications of cloud computing.

- Terminals: PC is not the only central computing device, various electronic devices including MP3, SmartPhone, Tablet, Set-top box, PDA, notebook are new terminals that have the requirement of personal computing. Besides, repeated data synchronization among different terminals is time-consuming so that faults occur frequently. In such cases, a solution that allows individuals to access to personal data anywhere and from any device is needed.

\section{Deployment models \& Service Models Deployment Models:}

As shown in fig 2 Clouds are deployed in different fashions, depending on the usage scopes. There are four primary cloud deployment models.

- Public cloud is the standard cloud computing paradigm, in which a service provider makes resources, such as applications and storage, available to the general public over Internet. Service providers charge on a fine-grained utility computing basis. Examples of public clouds include Amazon Elastic Compute Cloud (EC2), IBM's Blue Cloud, Sun Cloud, Google AppEngine and Windows Azure Services Platform.

- Private cloud looks more like a marketing concept than the traditional mainstream sense.It describes a proprietary computing architecture that provides services to a limited number of people on internal networks. Organizations needing accurate control over their data will prefer private cloud, so they can get all the scalability, metering, and agility benefits of a public cloud without ceding control, security, and recurring costs to a service provider. Both eBay and HP Cloud Start yield private cloud deployments.

- Hybrid cloud uses a combination of public cloud, private cloud and even local infrastructures, which is typical for most IT vendors. Hybrid strategy is proper placement of workloads depending upon cost and operational and compliance factors. Major vendors including HP, IBM, Oracle and VMware create appropriate plans to leverage a mixed environment, with the aim of delivering services to the business. Users can deploy an application hosted on a hybrid infrastructure, in which some nodes are running on real physical hardware and some are running on cloud server instances.

- Community cloud overlaps with Grids to some extent. It mentions that several organizations in a private community share cloud infrastructure. The organizations usually have similar concerns about mission, security requirements, policy, and compliance considerations. Community cloud can be further aggregated by public 
cloud to build up a cross-boundary structure.

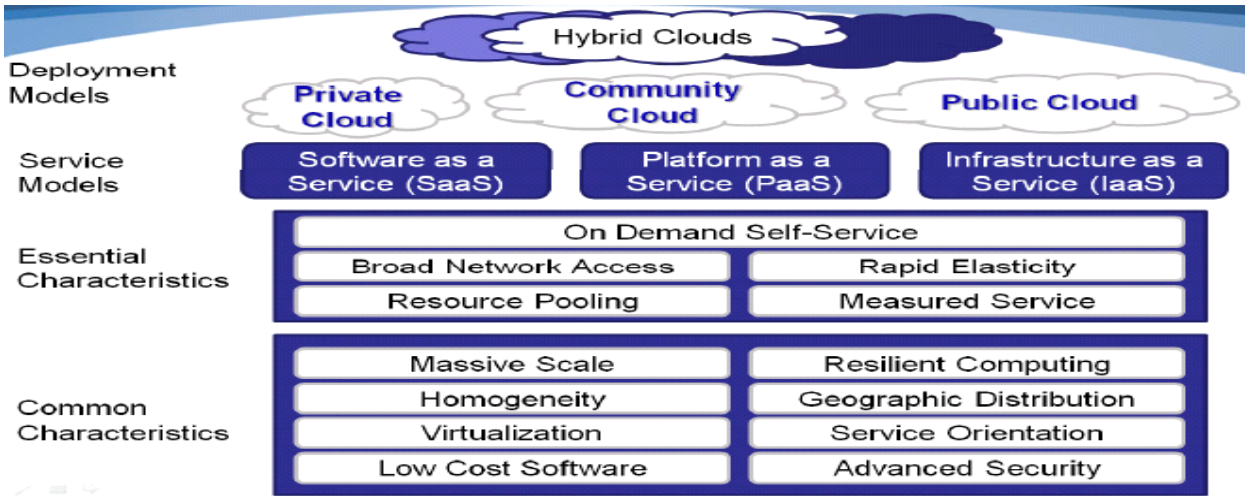

Figure 2 - Cloud Deployment and Service Models

\section{Service Models}

As shown in Fig-2 an underlying delivery mechanism, cloud computing ability is provisioned as services, basically in three levels: software, platform and infrastructure .

\section{Software as a Service}

Software as a Service (SaaS) is a software delivery model in which applications are accessed by a simple interface such as a web browser over Internet. The users are not concerned with the un derlying cloud infrastructure including network, servers, operating systems, storage, platform,etc. This model also eliminates the needs to install and run the application on the local computers. The term of SaaS is popularized by Salesforce.com, which distributes business software on a subscription basis, rather than on a traditional on-premise basis. One of the best known is the solution for its Customer Relationship Management (CRM). Now SaaS has now become a common delivery model for most business applications, including accounting, collaboration and management. Applications such as social media, office software, and online games enrich the family of SaaSbased services, for instance, web Mail, Google Docs, Microsoft online, NetSuit, MMOG Games, Facebook, etc.

\section{Platform as a Service}

Platform as a Service (PaaS) offers a high-level integrated environment to build, test, deploy and host customer-created or acquired applications. Generally, developers accept some restrictions on the type of software that can write in exchange for built-in application scalability. Customers of PaaS do not manage the underlying infrastructure as SaaS users do, but control over the deployed applications and their hosting environment configurations.

PaaS offerings mainly aim at facilitating application development and related management issues. Some are intended to provide a generalized development environment, and some only provide hosting-level services such as security and on-demand scalability. Typical examples of PaaS are Google App Engine, Windows Azure, Engine Yard, Force.com, Heroku, MTurk.

\section{Infrastructure as a Service}

Infrastructure as a Service (IaaS) provides processing, storage, networks, and other fundamental computing resources to users. IaaS users can deploy arbitrary application, software,operating systems on the infrastructure, which is capable of scaling up and down dynamically. IaaS user sends programs and related data, while the vendor's computer does the computation processing and returns the result. The infrastructure is virtualized, flexible, scalable and manageable to meet user requirements. Examples of IaaS include Amazon EC2, VPC, IBM Blue Cloud, Eucalyptus, FlexiScale, Joyent, Rackspace Cloud, etc.

\section{Related Technologies:}

Although the term 'cloud computing' is relatively new, the technologies that make it possible have been used for some time. For example, cloud computing is enabled by grid computing, virtualization, utility computing, hosting and software as a service (SaaS). Yet these technologies differ from cloud computing in the following ways:

\section{Grid Computing:}

Originally designed for computationally intensive batch applications, Grid computing was developed as a way to share computers and data. From these beginnings, modern Grid computing technologies have 
evolved as a way to harness inexpensive servers in a data center to solve a variety of business problems. Traditionally, grids have lacked the automation, agility and simplicity characterized by cloud computing.

\section{Virtualization:}

Virtualization is a framework or methodology of dividing the resources of a computer into multiple execution environments, by applying one or more concepts or technologies such as hardware and software partitioning, time-sharing, partial or complete machine simulation, emulation, quality of service, and many others. It allows abstraction and isolation of lower-level functionalities and underlying hardware. This enables portability of higher-level functions and sharing and/or aggregation of the physical resources. There are lots of virtualization products, and a number of small and large companies that make them. For instance, in the operating systems and software applications space are VMware1, Xen - an open source Linux-based product developed by XenSource2, and Microsoft virtualization products, can be mentioned.

Virtualized infrastructures are available on-demand and capable of supporting multiple users, but lack the automation required for the self-managing, self-healing property of the cloud.

Hosting: Hosting services provide space on servers for use by clients as well as providing IP-based connectivity, typically in a data center. Because hosting does not enable on-demand, elastic scalability, it cannot be considered cloud computing.

SaaS: Although Software-as-a-Service offerings are often hosted on true clouds, SaaS is an application as a service and cloud computing is IT infrastructure as a service.

Utility computing: In the utility computing model, computing resources like applications, infrastructure and storage are packaged and sold as a service, with users paying only for what they consume, like electricity. In many respects, cloud computing is closely related to the utility computing model, with cloud computing being a broader concept that relates to the underlying architecture in which the services are designed.

\section{Drivers for Cloud Adoption:}

Cloud computing represents a convergence of several IT drivers and offers cost-effective solutions to key business demands. For example, clouds provide businesses with the agility required to move quickly in highly competitive business environments. This allows organizations to activate and retire resources as needed, dynamically update infrastructure elements, and move workloads to improve efficiency without having to worry about creating new infrastructures for each new application.

Organizations want to take advantage of several cost benefits provided by cloud computing. These include the price/performance offered by readily-available, commodity-grade computers, the ability to mitigate skyrocketing data center development and operational costs and utilizing a shared infrastructure rather than creating new platforms on an application-by-application basis.

\section{How Enterprises Are Using the Cloud:}

Forward-thinking enterprises are using the cloud within their private data centers to take advantage of the best practices that public clouds have established, namely scalability, agility, automation, and resource sharing. Creating these private clouds enable IT departments to focus on innovation for the business, reducing both capital and operational costs and automating the management of complex technologies .

Moreover, the core applications within technology-driven enterprises create the most strategic competitive advantages. Because each enterprise has unique challenges, these are typically large-scale customdeveloped applications whose development, deployment and management can greatly benefit from a cloudenabled platform. It is for this reason that the financial services industry is among to venture down the path towards private cloud computing path though its deployment of grids, virtualization, and utility computing to organize internal IT resources.

\section{The Cloud Application Platform:}

To clearly appreciate the benefits of cloud computing for businesses, it is important to distinguish between the promise of the cloud and necessity of a cloud-enabled application platform. Ultimately, the greatest advantages of cloud computing can't be realized if an organization's applications are unable to take advantage of the cloud's inherent flexibility. For example, just because an organization is running applications on Amazon's EC2 or employing virtualization does not mean those applications can scale like amazon.com. In order to take advantage of the scalability, agility and reliability of the cloud, applications, applications must be built on a cloud-enabled platform. 
Building truly robust applications that make it easy for organizations to take advantage of the cloud's characteristics is a difficult, time-consuming task that can severely tax an organization's resources. Cloud- based application platforms dramatically simplify the delivery of cloud-enabled applications by abstracting the complexity and dynamic nature to quickly and inexpensively bring new capabilities to market, with the agility, reliability and scale demanded by their businesses.

\section{Summary \\ Pros \& Cons of Cloud \\ Pros:}

- $\quad$ Reduced Cost: Cloud technology is paid incrementally (you pay only for what you need), saving organizations money in the short run. Money saved can be used for other important resources.

- Increased Storage: Organizations can store more data than on private computer systems.

- Highly Automated: IT personnel not needed to keep software up to date as maintenance is the job of the service provider on the cloud.

- More Mobility: Employees can access information wherever they are, rather than having to remain at their desks.

- Allows IT to Shift Focus: No longer having to worry about constant server updates and other computing issues, government organizations will be free to concentrate on innovation.

Cons:

GNU founder Richard Stallman says that the interesting thing about cloud computing is that we've redefined cloud computing to include everything that we already do. One reason you should not use web applications to do your computing is that you lose control. It's just as bad as using a proprietary program . But certainly shifting to cloud computing has other problems including:

- Security: Is there a security standard?

- Reliance on 3rd Party: Control over own data is lost in the hands of an "difficult-to-trust" provider

- Cost of transition: Is it feasible for me to move from the existing architecture of my data center to the architecture of the cloud?

- Uncertainty of benefits: Are there any long term benefits?

\section{Conclusion:}

Industry analysts including Gartner and Forrester are early proponents of cloud computing and it's potential. Several trends are emerging that will enable enterprises to make good use of cloud computing, such as shared, virtualized and automated IT architectures. However, the introduction of cloud-enabled application platforms will certainly accelerate cloud adoption among businesses of all sizes.

\section{Acknowledgment}

I would like to thank my guide Prof. Altaf B. Mogal who has supported me a lot for my research work.

\section{References}

[1] J. Geelan, "Twenty-one experts define cloud computing,"Cloud Computing Journal, vol.4, pp. 1-5, 2009.

[2] "Cloud Computing - Issues, Research and Implementations" Mladen A. Vouk Department of Computer Science, North Carolina State University, Raleigh, NC 27695, USA Proceedings of the ITI $200830^{\text {th }}$ Int. Conf. on Information Technology Interfaces, June 23-26, 2008, Cavtat, Croatia

[3] "Cloud computing for small lresearch groups in computational science and engineering: current status and outlook "Computing(2011) (C) Springer-Verlag2010 